\title{
CORRELATION BETWEEN HEATS OF DEPOLYM- ERIZATION AND ACTIVATION ENERGIES IN THE DEGRADATION OF POLYMERS
}

\author{
Introduction
}

Detailed literature on thermal degradation of various synthetic organic polymers are available wherein activation energy $(E)$ values, degradation products, and possible mechanism of degradation process have been presented. However, the relevance of $E$ values with respect to the thermochemical properties of monomers and polymers has not received much attention.

On thermal initiation, polymer degradation can result both in depolymerization, producing gaseous products, and/or crosslinking, which produces an involatile char. Examples where depolymerization results only in gaseous products are addition polymers like polystyrene. Petrella and Sellars have shown that the pyrolysis of polystyrene is similar to that of styrene monomer (1). By similarity of polystyrene and styrene monomer, it is meant that the final pyrolysis products of both in the high-temperature region are the same. The reason for this is that polystyrene initially degrades to produce styrene monomer, which on subsequent steps gives various pyrolytic products. Further, the same authors have shown by flash pyrolytic combustion that the first step in the combustion of polystyrene involves depolymerization to monomer which undergoes different subsequent steps. Recently, Kishore et. al (2) have also shown that styrene is formed during the pyrolysis in the temperature range of $350-400^{\circ} \mathrm{C}$. As the heats of polymerization data and the $E$ values for the depolymerization of such polymers are readily available, one can see how the heats of depolymerization and $\mathrm{E}$ values are correlated. The purpose of the present paper, therefore, is to see whether there exists such a correlation in those addition polymers which on degradation yield only gaseous products. It may be noted that we are concerned here with the low-temperature region, where the degradation leading to volatile formation starts taking place.

\section{Results and Discussion}

On the basis of the assumption that the enthalpy change in the formation of solid polymer from gaseous monomer is equal to the enthalpy change during the breaking of the solid polymer into gaseous monomer, the values for the heats of depolymerization of various polymers into gaseous monomers have been calculated (3) and are presented in Table I. In those cases where the data for heats of polymerization for only liquid monomer to solid polymer are available, the heats of vaporization of liquid monomer (4) to gaseous monomer have been added. The E values (Table I) have been cited from literature. 


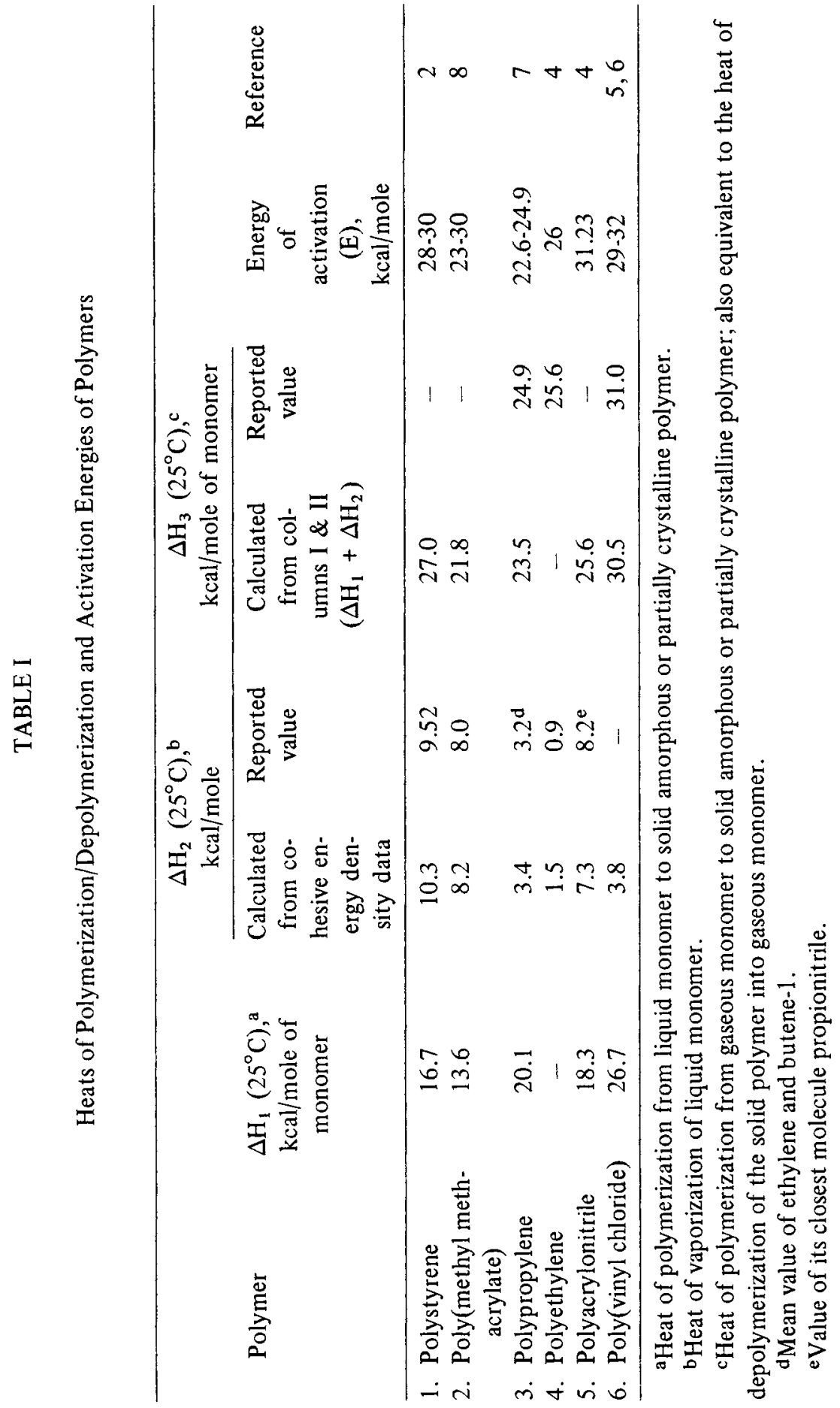


Table I clearly shows that heats for depolymerization of the polymers are almost same as their respective activation energies, suggesting thereby that degradation takes place in the following way:

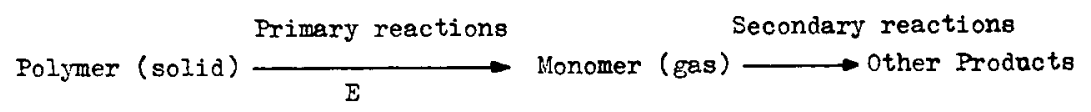

The above scheme is definitely a simplified one because it is well known. that polymer is not directly converted into monomer; instead, it involves mainly two steps: (i) the initiation to form macroradicals and (ii) depropagation to give monomer. The agreement between the calculated heats of depolymerization to yield gaseous monomer and the experimental $E$ values suggests two things. Firstly, the above two steps proceed with same rapidity during the degradation, and therefore the $E$ value is a combination of the energy required for the occurrence of above two processes. Alternatively, one of the two steps (most probably the initiation step) is slower and requires much more energy compared to the other; therefore, the net energy corresponding to the $\mathrm{E}$ for the total process is equal to the energy required for this slower process. It is well known that depropagation requires much less energy. The authors feel that the most likely proposition is that initiation is the rate-determining reaction and requires most of the energy during the conversion of polymer to monomer.

The conversion of polymer into gaseous monomer is the rate-controlling step, and the latter decomposes and/or reacts with secondary products to give various degradation species. At higher temperatures, due to the fact that kinetic chain lengths may be larger than the chain lengths of macroradicals formed, the degradation behavior may be entirely different than at lower temperatures. However, as was pointed out earlier, our present analysis is limited only to the low-temperature degradation reactions. It may also be possible that at higher temperatures the degradation reactions may become quite complex so that the rate-controlling step may also change. Such behavior has been observed in some polymers.

It may be noted that for polyethylene and polypropylene, quite a few $\mathrm{E}$ values cited in literature are higher than reported in Table I. The reason for this difference is discussed below.

On thermal treatment, in the absence of oxygen, these polymers first undergo cyclization or crosslinking. As the temperature is increased, scission takes place forming satured hydrocarbons, like ethane, propane, or n-pentane, together with the monomer. (A mixture of ethylene and propylene is obtained in both polymers.) The monomer formation occurs via depolymerization or a cyclic process. The larger formation of monomer can be achieved by a careful selection of experimental conditions (9). A better yield of monomer formation can be expected for branched polymers compared to the unbranched ones, (10) and hence the activation energy (E) calculation would favor branched polymers of low molecular weights (5). It may be noted that the unbranched polymers are more thermally stable than the branched ones (11). 
This is to note that literature on thermal degradation of polystyrene shows a high variation in the $\mathrm{E}$ values ranging from 20 to $83 \mathrm{kcal} / \mathrm{mole} \mathrm{(2).} \mathrm{We} \mathrm{have}$ recently shown by using different techniques and different equations that an erroneous value of $E$ could result from the choice of an unsuitable equation. Further work will be needed to see whether the wide variation in the $E$ value from the thermal decomposition of polyethylene and polypropylene arises due to inappropriate use of equations to calculate $E$ values. In the case of polystyrene, we have shown that the $\mathrm{E}$ values remain the same for oxidative as well as nonoxidative thermal degradation of polystyrene. One would thus expect a similar trend for the polyethylene-polypropylene system. If that is the case, then our calculated value (Table I) comes pretty close to the value of E 27 for the thermooxidative degradation of $\alpha$-olefinic polymers (11). Whether the activation process for thermooxidative degradation is the same as that of pure thermal degradation has to be verified by more experimental results. However, in the case of polystyrene, we have shown that the activation process in both thermal and thermal oxidative degradation is the same (2).

Significant mechanistic differences exist between polyacrylonitrile (PAN) and poly(vinyl chloride) (PVC) on one hand and the remainder on the other. Based on the mechanism that PVC on degradation gives $\mathrm{HCl}$ and carbon-containing residue and PAN degrading into $\mathrm{HCN}$ and carbon-containing residue, we have carried out enthalpy calculations; the resulting values are $13.3 \mathrm{kcal} /$ mole and $19.4 \mathrm{kcal} / \mathrm{mole}$, respectively. The values are not in agreement with the reported $E$ values of such polymers (Table I). The close agreement between the reported $E$ values and our calculated values (calculation based on the consideration that in the rate-limiting process the solid polymer is converted to gaseous monomer) makes us believe that the rate-determining step in the thermal decomposition of PVC and PAN is the formation of the gaseous monomer from the polymer. These monomers, however, being very unstable due to the presence of $-\mathrm{Cl}$ and $-\mathrm{CN}$ groups, decompose rapidly into $\mathrm{HCl}$ and $\mathrm{HCN}$, respectively, and the residue. We therefore checked the literature to search for the evidence of the formation of monomer fraction in the degradation products of PVC and PAN. The formation of monomer amongst the degradation products of PAN has been reported (12).

It may well be possible on one hand that our basic thesis does not apply to the degradation of polymers which do not monomerize prior to the degradation. On the other hand, the rate-determining step, as pointed out earlier in the case of poly(vinyl chloride) and polyacrylonitrile, may still be the polymer first degrading to give gaseous monomer, and we may be able to apply this concept to variety of polymers.

To probe further for the clarification of these points, more kinetic and thermochemical data for various polymers are needed.

\section{References}

(1) R. V. Petrella and G. D. Sellers, Combust. Flame, 16, 83 (1971).

(2) K. Kishore, V. R. Pai Verneker, and M. N. R. Nair, J. Appl. Polym. Sci., in press. 
(3) J. Brandrup and E. H. Immergut, "Polymer Handbook," Interscience, New York, 1966.

(4) J. Timmermans, "Physico-Chemical Constants of Pure Organic Compounds," Vol. 2, Elsevier, Amsterdam, 1965.

(5) S. L. Madrosky "Thermal Degradation of Organic Polymers," Interscience, New York, 1964, pp. 124, 196, 197.

(6) G. C. Marks, J. L. Benton, and C. M. Thomas, Soc. Chem. Ind. (London), Monogr. No. 25, 204-31, Disc. $231-5$ (1967); Chem. Abstr., 68, 30349 (1968).

(7) Z. Osawa, Shirku Kagaku, 14(1), 2 1966; Chem. Abstr., 68, 30350K (1968).

(8) H. H. G. Jellinek and M. D. Luh, Makromol. Chem., 115, 89 (1968);

Chem. Abstr., 69, 36571 a (1968); ibid., 66, 105, 329e (1967).

(9) A. W. J. Baily and C. Liotta, Amer. Chem. Soc. Div. Polym. Chem. Prepr., 5(2), 333 (1964).

(10) R. T. Conley, "Thermal Stability of Polymers," Vol. 1, Dekker, New York, 1970 pp. 298-299.

(11) L. Reich and S. S. Stivala, "Elements of Polymer Degradation," McGrawHill Book Co., New York, 1971, pp. 298, 170.

(12) F. A. Bell, R. S. Lehrle, and J. C. Roble, Polymer, 12, 579 (1971).

\section{K. Kishore* \\ V. R. Pai Verneker}

High-Energy Solids Laboratory

Department of Inorganic and Physical Chemistry

Indian Institute of Science

Bangalore 560 012, India

Received December 5, 1975

Revised July 30, 1976

*Author to whom all communications should be addressed. 